\title{
XXXIX. Optische Studien an pseudosymmetrischen Krystallen.
}

\author{
Von \\ G. Wulff aus Warschau*). \\ (Mit 4 Holzschnitten.)
}

\section{Das Berylliumsulfat $\left(\mathrm{BeSO}_{4}+4 \mathrm{H}_{2} \mathrm{O}\right)$.}

Das Material, das ich für meine Studien an diesem Salze verwendet habe, war vorher von Dr. F. Grunling aus Phenakit dargestellt und war noch durch Gyps und Eisenoxydulsulfat verunreinigt. Die Trennung der Sulfate geschah durch Behandeln der gefällten Hydrate mit Ammoniumcarbonat. Als ich die ziemlich gesättigte Lösung des so erbaltenen Berylliumsalzes längere Zeit bei constanter Temperatur (im Keller) ubber Schwefelsäure krystallisiren liess, erhielt ich bis ein Centimeter grosse, farblose, durchsichtige Krystalle von derselben Form, wie sie bereits von Topsö ${ }^{* *}$ ) beschrieben worden ist: es waren nämlich meist verzerrte tetragonale Pyramiden, combinirt mit dem Prisma zweiter Ordnung. Wenn wir die Aufstellung von Topsöe annehmen, so stellen die Krystalle die Combination von $\{101\}$ und $\{110\}$ dar; die letztere Form fehlte zuweilen. Die Flächen von $\{101\}$ waren so unregelmässig gekrummt, dass man in den meisten Fällen kein gutes Bild im Gesichtsfelde des Goniometers bekommen konnte und daher die einzelnen Messungen um einige Grade verschieden waren. Ich erhielt für den Winkel zwischen den Flächen von $\{101\}$ in der horizontalen Zone Werthe, welche zwischen $90^{\circ}$ und $93^{\circ}, 5$ schwankten $\left.{ }^{* *}\right)$. Neben einfachen, unregelmässig mit einander verwachsenen Krystallen bilden sich manchmal höchst eigenthümliche, regelmässige Verwachsungen, be-

*) Die vorliegende Untersuchung ist im mineralogischen Laboratorium der k. Akademie der Wissenschaften zu München ausgeführt.

**) Sitzungsber. d. k. Akad. d. Wiss. zu Wien 1872.

***) Nach $\mathrm{T}$ op sö e soll dieser Winkel $93010^{\prime}, 5$ betragen. 
stehend aus vier neben einander in paralleler Stellung auf der Basis liegenden Pyramiden, während die vier Zwischenräume zwischen deron gegen einander geneigten Flächen ausgefüllt werden durch zwei Paare eben solcher Pyramiden, wobei aber die Hauptaxen der letzteren horizontal in zwei auf einander senkrechten Richtungen verlaufen. Fig. 1 soll die Erscheinung in

Fig. 1.

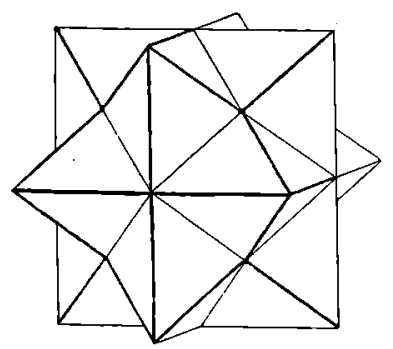

Fig. 2.

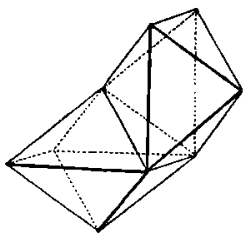

Fig. 3.

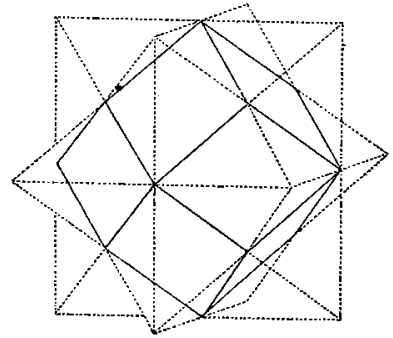

vollständiger Form darstellen. In der Wirklichkeit bildet sich der untere (in der Figur hintere) Theil nicht aus, weil die Pyramiden auf der Basis als Auflagerungsfläche sich bildeten. Bei leichtem Andrücken zerfällt zuweilen das Gebilde in einzelne Pyramiden. Wenn wir dieses Gebilde rein geometrisch betrachten, so mussen wir hier ohne weiteres das Rhombendodekaëder erkennen, welches aber in jedem Oktanten eine sechskantige einspringende Ecke besitzt. Mit anderen Worten: man kann sich dieses Polyëder aus dem Rhombendodekaëder entstanden denken, indem man sämmtliche Flächen bis zum gegenseitigen Schnitte verlängert*).

Wenn wir uns der Mallard'schen Annahme anschliessen, dass die pseudoregulären Formen Zwillingsbildungen sind, so müssen wir fưr solche Fälle die Möglichkeit von regulären Formen mit einspringenden Winkeln zulassen und somit die hier vorliegende eigenthumliche Art des Rhombendodekaëders als eine unmittelbare Folge dieser Annabme betrachten.

Wurde man sechs solcher tetragonaler Pyramiden so aneinander legen, dass ihre Hauptaxen sich in einem Punkte schneiden, so würde daraus, wie durch Vergleichung von Fig. 2 und 3 leicht einzuseben, ein Rhombendodekaëder sich zusammensetzen und somit die Symmetrie des regulären Systemes durch die Wiederholung des einfachen Verwachsungsgesetzes : Zwillingsebene einer Fläche der Pyramide selbst nacbgeahmt. Solche

*) Fig. 2 zeigt zwei Elemente des ganzen Gebildes, welche nach (101) verzwillingt sind, und Fig. 3 veranschaulicht die Entstehung des Gebildes aus einem Rhombendodekaëder. Alsdann ist die Form der Pyramide des Berylliumsulfates, deren Winkel, wie wir oben sahen, in der Nähe von 900 schwankende sind, als eine "Grenzform “ im Malla rd'schen Sinne zu betrachten, deren idealer Winkel genau 900 betrüge.

Groth, Zeitschrift f. Krystallogr. XVII. 
Rhombendodekaëder ohne einspringende Winkel sind von mir nicht beob. achtet worden.

Die Substanz der Krystalle ist stark doppeltbrechend. Die directo Messung der Brechungsindices mit einem sehr sorgfältig geschliffenen Prisma hat folgende Werthe ergeben:

$\begin{array}{ccc}\text { Linie : } & \omega & \varepsilon \\ C & 1,4686 & 1,4299 \\ D & 1,4714 & 1,4322 \\ F & 1,4769 & 1,4367\end{array}$

Die Doppelbrechung ist also negativ.

Wenn wir einen Schliff aus dem Krystalle senkrecht zur Hauptaxe anfertigen, so erblicken wir im convergenten Lichte sehr unregelmässige Ringe, welche manchmal die Gestalt von zwei sich senkrecht durchkreuzenden 8 haben. Stat eines schwarzen Kreuzes nimmt man öfters eine ganze Reihe. buntgefärbter wahr, die gegen einander verschiedenartig geneigt sind. Wenn man die Platte weiter schleift, so zeigt sie verschiedenartige bunte Flecke, welche theils gerad-, theils krummlinig begrenzt und manchmal uber die ganze Platte vertheilt sind. Die Platte wird zwischen gekreuzten Nicols nur an einigen Stellen stetig dunkel, welche zugleich als homogen einaxig erscheinen.

Eine ziemlich dünne Platte lässt die Gesetzmässigkeit der Erscheinung constatiren. Bei der Dicke von ungefähr $0,3 \mathrm{~mm}$ bemerkt man auf einem homogenen einaxigen Grunde zwei aufeinander senkrechte Systeme von parallel begrenzten Streifen, die stark doppeltbrechend sind und allerlei Farben der ganzen Newton'schen Scala zeigen, wobei derselbe Streifen oft seine Farbe stetig oder sprungweise ändert. Falls ein Streifen Farben höherer Ordnung besitzt, so ist er immer an seinen Rändern mit farbigen Fransen gesäumt. Die optischen Hauptschnitte der Streifen verlaufen parallel und senkrecht zu den Rändern derselben. Die den längeren Dimensionen des Streifens parallele Schwingung hat grössere Geschwindigkeit, wie die Prufung mit der Gypsplatte ergab. Es ist nur e in e Erklärung fưr die Natur dieser Streifen möglich: wir müssen annehmen, dass die Streifen Zwillingslamellen nach (101) sind. Das ergiebt sich aus folgenden Ueberlegungen.

1) Die scheinbare Breite aller Streifen in demselben Schliffe ist gleich.

\begin{tabular}{lcccc} 
& \multicolumn{4}{c}{ Breite in Theilen des Mikrometers. } \\
Schliff I. & $\mathbf{3 , 3 7}$ & $\mathbf{3 , 4 2}$ & $\mathbf{4 , 6 0}$ & $\mathbf{4}, 67$ \\
Schliff II. & $\mathbf{0 , 8 4}$ & $\mathbf{0 , 9 0}$ & $\mathbf{0 , 7 6}$ & 0,70
\end{tabular}

Der Unterschied zwischen den Angaben ist durch die ungleichmăssige Dicke der Schliffe verursacht.

2) Die Streifen verlaufen in dem Schliffe schräg von oben nach unten, 
so dass die beiden Ränder nicht zugleich im Mikroskope scharf sichtbar sind. Wenn wir die Dicke des Schliffes und die scheinbare Breite der Streifen messen, so können wir den Winkel, den die letzteren mit der Richtung des Schliffes einschliessen, bestimmen. Dieser Winkel beträgt ungefähr $45^{\circ}$, was der Neigung der Flächen $\{101\}$ und $\{001\}$ entspricht.

3) Die Abstände zwischen den Fransen können in Betracht gezogen werden, um daraus die Doppelbrechung der Streifen in der Richtung der Kante [001] zu berechnen. Diese Messungen habe ich mit besonderer Sorgfalt ausgefüht, indem ich bei verschiedenen Vergrösserungen die Abstände der Fransen mit dem Schraubenmikrometer am Mikroskope von Fuess, Mod. Nr. 1, mass. Wenn man das obige Zwillingsgesetz annimmt, so sollte die Grösse dieses Abstandes 0,0140 mm betragen. Aus den directen Messungen habe ich den Werth $0,0135 \mathrm{~mm}$ bekommen. Das beweist also, zusammen mit den oben angefuhrten Grunden, dass diese Streifen nichts anderes sind, als Zwillingslamellen des Berylliumsulfates nach dem oben nachgewiesenen Gesetze.

Wenn wir die Zwillingslamelle im polarisirten, convergenten Lichte betrachten, so erscheint sie zweiaxig, was theoretisch der Fall sein muss, da hier eine düne doppeltbrechende Lamelle sich zwischen zwei einaxigen, d. $h$. in der Richtung der Axe des Instrumentes einfach brechenden Medien befindet. Der Axenwinkel nimmt zu mit der Zunahme der Dicke der Zwillingslamellen. Bei derjenigen Dicke der letzteren, bei welcher Interferenzfarben höherer Ordnungen erzeugt werden, sind die schwarzen Hyperbeln nicht mebr wahrnehmbar.

Die Schliffe, welche ich nach anderen Richtungen verfertigt habe, bieten nichts Bemerkenswerthes, sie haben nur das bestätigt, was ich in den oben beschriebenen Schliffen gesehen habe.

\section{Das wasserfreie Kalium-Lithium-Doppelsulfat $\left(\mathrm{Ka} \mathrm{LiSO} \mathrm{O}_{4}\right)$.}

Obgleich die Krystalle dieses Salzes schon mehrmals beschrieben worden sind, so hat man doch manche sehr interessante Eigenschaften desselben ubersehen. Herr Prof. P. Groth gab mir einige Krystalle zur Untersuchung, welche nach der Etiquette Lithion-haltiges schwefelsaures Kalium sein sollten und sich als das obige Doppelsulfat erwiesen. Da ich daran manche neue Eigenschaften entdeckte, wie z. B. die Gircularpolarisation, so unternahm ich, das Salz selbst darzustellen. Saures schwefelsaures Kalium wurde zu diesem Zwecke mit kohlensaurem Lithium gesättigt und in den Krystallisationskeller über Schwefelsäure gestellt. Es bildeten sich zuerst Krystalle von Kaliumsulfat, welche ich von Zeit zu Zeit aus der Lösung entfernte. Dann trat in der Krystallisation ein Ruhezustand ein, wonach prachtvolle, durchsichtige, grosse Krystalle von $\mathrm{Ka} \mathrm{LiSO}_{4}$ ausgeschieden wurden. 
Die Form der Krystalle war die Combination $\{0001\},\{10 T 1\}$ und $\{10 T 0\}$. Alle Krystalle zeigten in der verticalen. Zone beträchtliche Schwankungen in der Grösse der Winkel und in der Parallelität der Flächen. Aus meinen Messungen ergab sich, dass die mittlere Schwankung der Grösse des Prismenwinkels $\pm 25^{\prime}$, diejenige der Parallelität $\pm 23^{\prime}$ beträgt, was ich nach der Methode der kleinsten Quadrate erhielt. Man sieht, dass beide Grössen fast einander gleich sind. Für den Winkel zwischen den Flächen von $\{10 T 1\}$ und $\{0001\}$ erhielt ich aus mehreren Messungen den Werth 620 39' $\pm 3^{\prime}$, wobei die mittlere Schwankung dieses Winkels $\pm 14^{\prime}$ gefunden worden ist. Aus diesen Angaben ergab sich das Axenverbältniss :

$$
a: c=0,597 \pm 0,001 \text {. }
$$

Die Stellung der Krystalle im hexagonalen Systeme wird später besprochen.

Die Brechungsindices wurden nach der Prismenmethode bestimmt. Ich bediente mich dazu des Websky'schen Spaltes, und da die Doppelbrechung sehr gering war, so deckten die breiten Theile des Bildes theilweise einander. Das Fadenkreuz des Fernrohres wurde auf die Mitte des so entstandenen gemeinschaftlichen Bildes eingestellt. Dann habe ich noch die Winkelabstände der einzelnen Bilder von einander gemessen. Dieses Verfahren wendete ich dazu an, um gleichzeitig auch die Grösse der Doppelbrechung etwas genauer zu bestimmen. Es sei nämlich $2 \varepsilon$ der Abstand der beiden Bilder im Winkelmaasse, $\varphi$ der Prismenwinkel und $\delta$ die Ablenkung der Mitte des doppelten Bildes, so sind die beiden Brechungsindices*)

oder, weil $\varepsilon$ klein ist,

$$
\begin{aligned}
& n_{1}=\frac{\cos \frac{1}{2}(\varphi-\delta+\varepsilon)}{\cos \frac{1}{2} \varphi} \\
& n_{2}=\frac{\cos \frac{1}{2}(\varphi-\delta-\varepsilon)}{\cos \frac{1}{2} \varphi}
\end{aligned}
$$

$$
\begin{aligned}
& n_{1}=\frac{\cos \frac{1}{2}(\varphi-\delta)}{\cos \frac{1}{2} \varphi}-\frac{1}{2} \varepsilon \frac{\sin \frac{1}{2}(\varphi-\delta)}{\cos \frac{1}{2} \varphi}, \\
& n_{2}=\frac{\cos \frac{1}{2}(\varphi-\delta)}{\cos \frac{1}{2} \varphi}+\frac{1}{2} \varepsilon \frac{\sin \frac{1}{2}(\varphi-\delta)}{\cos \frac{1}{2} \varphi},
\end{aligned}
$$

woraus, wenn wir die Differenz $n_{2}-n_{1}$ durch $\Delta$ bezeichnen:

$$
\Delta=\varepsilon \frac{\sin \frac{1}{2}(\varphi-\delta)}{\cos \frac{1}{2} \varphi}
$$

Diese Grösse kann man in dem betrachteten Falle fast unabhängig von der etwaigen Krümmung der Prismenflächen erhalten.

*) Ich gebe den Formeln obige Gestalt, weil $\varphi$ hier den Winkel der Flächennormalen bedeutet. 
In solcher Weise wurden für die Spectrallinien $C, D$ und $F$ folgende Werthe erhalten :

$\begin{array}{lccc} & C & D & F \\ \omega & 1,4697 & 1,4715 & 1,4759 \\ \varepsilon & 1,4703 & 1,4721 & 1,4762 \\ \Delta & 59 \times 10^{-5} & 57 \times 10^{-5} & 31 \times 10^{-5}\end{array}$

Die Doppelbrechung wurde noch nach einer anderen und viel genaueren Methode bestimmt. Dasselbe Prisma, welches fur die obigen Messungen diente, zeigle im Polarisalionsmikroskope trotz seines grossen Winkels von $54^{\circ} 21^{\prime}$ sebr breite Interferenzfransen. Aus der Messung des absoluten Abstandes zweier benachbarter. Streifen im Natriumlichte konnte ich die Doppelbrechung berechnen und erhielt so :

$$
\Delta_{D}=591 \times 10^{-6} \text {, }
$$

was ziemlich nahe dem weniger genauen Werthe $57 \times 10^{-5}$ kommt. Dabei soll erwähnt werden, dass die Messungen bei der Temperatur von $24^{\circ}$ vollzogen wurden, weil, wie es später auseinander gesetzt wird, die Doppelbrechung sich mit der Temperatur merklich ändert.

Die Drehung der Polaris ationsebene war in manchen Krystallen sebr regelmässig, wenn diese selbst ganz homogen waren; in den truben Krystallen habe ich immer beträchtliche Störungen der Erscheinung beobachtet. Ich habe fur genauere Messungen nur ein Exemplar verwendet, weil es sich als vollständig homogen erwies. Die empfindlichste Methode, die mir das Mikroskop bei seinem jetzigen Zustande anzuwenden gestattete, war die folgende. Nach Beseitigung des unteren Nicols wurde ein anderer so. in die Oeffnung des Tischchens eingeschoben, dass er durch den hervorspringenden oberen Rand seiner Hülse am Herunterfallen gehindert war. Auf denselben wurde noch eine Glasplatte aufgelegt und so mit den beiden Federn des Tischchens festgehalten, dass sie den Nicol und das Tischchen als ein unveränderliches System zusammenhielt. Oben war das B e r trand'sche Quarzocular eingesetzt. Wenn man jetzt das Gesichtsfeld mit homogenem Lichte beleuchtet, so erscheinen die vier Sectoren des Oculars so lange ungleich hell, bis man das Tischchen, welches jetzt mit dem Polarisator fest verbunden ist, in die nöthige Lage durch Drehung bringt. Diese Lage entspricht dem Nullpunkte. Wenn man jetzt die circularpolarisirende Substanz einschiebt, so werden die Sectoren bei einer anderen Lage des Tischchens gleiche Helligkeit annehmen. Die Differenz beider Einstellungen giebt den gewunschten Drehungswinkel. Es ist einleuchtend, dass man den Nullpunkt viel sicherer in weissem Lichte bekommt, als in monochromatischem, weil da der Unterschied der Färbung und nicht der der Helligkeit in Betracht kommt. Bei mir war der mittlere Fehler der einzelnen Einstellung in weissem Lichte $\pm 7^{\prime}$ und in monochromatischem 
$\pm 17^{\prime}$. In dieser Weise habe ich für den Drehungswinkel für die Dicke $1 \mathrm{~mm}$ folgende Werthe erhalten :

$\begin{array}{ll}\text { Rothes Glas } & 2 \% 6, \\ \text { Natriumlicht } & 2,8,\end{array}$

wobei der Fehler des Resultates $\pm 0,1$ beträgt. Die Messungen an den trüben Exemplaren ergaben für Gelb die Grösse von etwa $3^{0}$.

Es giebt sowohl rechts- wie linksdrehende Krystalle, doch habe ich vorwiegend die der ersten Art gefunden. Einmal wurde ein Zwilling nach der Basis beobachtet, in welchem man sehr deutlich die A i ry'schen Spiralen erblicken konnte.

Es wird sehr begreiflich, warum diese merkwurdige Eigenschaft bis jetzt vollständig unbemerkt blieb, wenn man in Erwägung zieht, dass die Krystalle meistens sehr dunn nach der Hauptaxe sind und deshalb die Erscheinung sich fast vollständig der Beobachtung entzieht.

Die Krystalle des in Rede stebenden Salzes sind noch in einer Beziehung bemerkenswerth : ihre Doppelbrechung nimmt bedeutend mit steigendel' Temperatur zu. Um diese Aenderung näher zu untersuchen, bediente ich mich desselben Prismas, welches zur Messung der Brechungsexponenten gedient hatte. Zu diesem Zwecke wurde dieses Prisma in den De s Cloiz ea ux'schen Erwärmungskasten eingebracht und daselbst mittelst eines passenden Halters festgehalten. Das Mikroskop wurde horizontal gestellt und der Kasten zwischen dem Objectiv und Tischchen auf einem speciell zu diesem $Z$ wecke verfertigtem Stative befestigt. Nachdem man das Gesichtsfeld mit gelbem Natriumlichte beleuchtet hatte, wurde mit dem Schraubenmikrometer der Abstand der Fransen bei einer gewissen Temperatur, welche durch zwei symmetrisch angebrachte Buns en' sche Brenner einige Zeit constant gehalten werden konnte, gemessen. Da der Abstand der benachbarten Interferenzstreifen der Grösse der Doppelbrechung umgekehrt proportional ist, so sollte man, um eine bequeme graphische Darstellung der Erscheinung zu erhalten, die reciproken Werthe der $\mathrm{Ab}$ stände auftragen. Da die Auftragung der direct erhaltenen Grössen unmöglich war, so habe ich die Ordinaten, welche eben die Grösse der Doppelbrechung darstellen sollten, $4^{-1} \times 10^{5}$ mal vergrössert und dadurch folgende Zahlen erhalten:

Temperaturen als

Abcissen genommen $\begin{array}{llllllllll}24^{\circ} & 39^{\circ} & 50^{\circ} & 80^{\circ} & 89^{\circ} & 105^{0} & 1120 & 125^{\circ} & 144^{0} & 147^{\circ}\end{array}$

$\begin{array}{lllllllllll}\text { Ordinaten } & 121 & 128 & 132 & 145 & 152 & 163 & 167 & 169 & 177 & 179\end{array}$

Es ist leicht zu bemerken, dass die Reihe der so aufgetragenen Punkte (siehe Fig. 4) einer Geraden ziemlich nahe kommt. Wenn wir diese Gerade nach der Methode der kleinslen Quadrate berechnen, so finden wir folgende Gleichung : 


$$
y=0,483 x+109,2 \pm 1,9 .
$$

Da, wie es oben gefunden ist, die Grösse der Doppelbrechung $d$ für $240591 \times 10^{-6}$ beträgt, und da die Ordinaten $4^{-1} \times 10^{5}$ mal vergrössert sind, so findet man nach einer einfachen Umrechnung, dass

$$
\Delta=535 \times 10^{-6}+236 \times 10^{-8} t,
$$

wo $t$ die Temperatur bezeichnet.

Um die Resultate der Rechnung mit den Angaben der directen Messung zu vergleichen, wollen wir bemerken, dass die Punkte, welche die Abstände der Fransen repräsentiren, auf einer gleichseitigen Hyperbel liegen

Fig. 4.

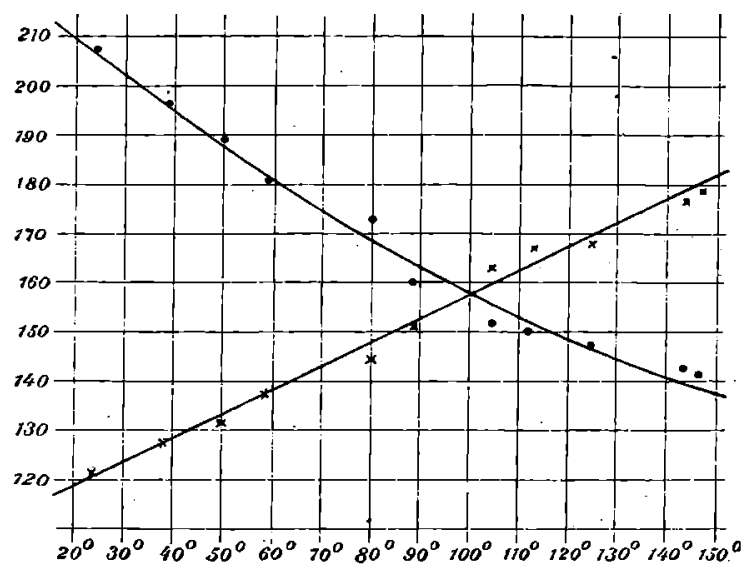

mussen, falls die reciproken Werthe der Ordinaten einer Geraden angehören. Wenn wir also statt $y$ in die Gleichung (1) $\frac{1}{n}$ setzen und dabei den Coordinatenanfang in den Punkt $y=0, x=-226,1$ übertragen, so bekommen wir folgende Gleichung:

$$
x n=51760 .
$$

Indem wir aus dieser Gleichung die Punkte der Hyperbel berechnen und sie mit den direct aufgetragenen Punkten vergleichen (Fig. 4), können wir die Resultate dieser Zusammenstellung durch folgende Tabelle ubersichtlich machen :

$\begin{array}{cccc}\text { Temperatur: } & \text { Berechnet: } & \text { Gemessen : } & \text { Fehler: } \\ 0^{0} & 229,0 & - & - \\ 10^{0} & 219,5 & - & - \\ 24^{0} & 207,0 & 207 & \pm 0 \\ 39^{\circ} & 195,3 & 195 & +0,3\end{array}$


G. Wulf.

$\begin{array}{cccc}\text { Temperatur: } & \text { Berechnet: } & \text { Gemessen: } & \text { Fehler: } \\ 50^{\circ} & 187,5 & 189 & -1,5 \\ 59^{0} & 481,5 & 181 & +0,5 \\ 80^{0} & 169,1 & 173 & -3,9 \\ 89^{0} & 164,3 & 160 & +4,3 \\ 105^{\circ} & 156,4 & 152 & +4,4 \\ 112^{0} & 153,1 & 150 & +3,1 \\ 125^{0} & 147,5 & 148 & -0,5 \\ 144^{\circ} & 140,0 & 143 & -3,0 \\ 147^{0} & 138,9 & 141 & -2,1\end{array}$

Es wurde schon fruher von $D$ u f e $t^{*}$ ) bemerkt, dass beim Beryll ebenfalls eine Zunahme der Doppelbrechung mit der Temperatur stattfindet, doch wird sie hier durch eine Parabel dargestellt und nähert sich einem Maximum, welches ungefähr der Temperatur $350^{\circ}$ entspricht.

In Folge der Gircularpolarisation ist anzunehmen, dass die Krystalle des $\mathrm{Ka} \mathrm{LiSO}_{4}$ einer der enantiomorphen Abtheilungen des hexagonalen Systemes angehören, und zwar der trapezoëdrisch-tetartoëdrischen, was auch durch Aetzfiguren, welche ich bier genauer zu untersuchen nicht die Absicht habe, bestätigt worden ist **). Es muss deshalb angenommen werden, dass das $\mathrm{K}_{2} \mathrm{SO}_{4}$ sowohl, als das $\mathrm{Li}_{2} \mathrm{SO}_{4}$ dimorph sein müssen, und zwar haben wir hier vollständige Analogie mit der Dimorphie des kohlensauren Kalkes, welche wir jetzt näher betrachten wollen.

\section{Zur Frage über die Dimorphie des kohlensauren Kalkes.}

Wenn wir annehmen, dass der Unterschied zwischen Galcit und Aragonit nur in der Art und Weise der Vertheilung derselben doppeltbrechenden Elemente besteht, so können wir ohne Weiteres auf diesen Fall die Mallard'sche Theorie anwenden und die Brechungsindices des Calcits aus denjenigen des Aragonits berechnen. Wenn wir die Brechungsindices des Aragonits mit $\alpha, \beta$ und $\gamma$ bezeichnen, wobei $\alpha>\beta>\gamma$, und diejenigen des Calcits mit $\omega$ und $\varepsilon$, so muss die Beziehung bestehen

$$
\omega=\frac{1}{2}(\alpha+\beta) ; \quad \varepsilon=\gamma .
$$

Wenn wir fur die in Betracht stehenden Grössen folgende Werthe annehmen: $\alpha=1,6859, \beta=1,6816, \gamma=1,5301, \omega=1,6585$ und $\varepsilon=$ 1,4863 , so erhalten wir die berechneten Werthe für $\omega$ und $\varepsilon$, die ich mit $\omega^{\prime}$ und $\varepsilon^{\prime}$ bezeichnen will,

$$
\omega^{\prime}=1,6837 ; \quad \varepsilon^{\prime}=1,5031,
$$

was sehr wenig der Beobachtung entspricht.

*) Bull. de la Soc. Min. de Fr. 1885, No. 7. S. diese Zeitschr. 12, 653.

**) Die Figuren waren zwar symmetrisch, doch hat ihre Vertheilung auf den Pyramidenflächen die Möglichkeit der trapezoëdrischen Hemiëdrie ausgeschlossen. 
Wir können abér durchaus nicht behaupten, dass diese Differenz zwischen Theorie und Wirklichkeit die Unanwendbarkeit der Theorie auf den betrachteten Fall zur Folge hat.

Wenn wir nur bedenken, dass die Entstehung beider Mineralien unter ungleichen Verhältnissen stattgefunden hat, so müssen wir bei der Berechnung noch die ubrigen Umstände in Betracht ziehen, welche ihren Grund in der Verschiedenheit der Entstehung haben. In erster Linie muss also die Dichtigkeit berucksichtigt werden, die beim Calcit 2,72 beträgt und beim Aragonit 2,94; doch ist es unmöglich die Correction der Brechungsindices in dieser Beziehung zu vollziehen, weil wir die Anordnung der Moleküle nach verschiedenen Richtungen in beiden Fällen nicht kennen. Wir können aber umgekehrt aus den Aenderungen der Indices die der Dichte berechnen, wie aus Folgendem hervorgeht.

Betrachten wir der Bequemlichkeit wegen den ganz speciellen Fall der Formel, welche Malla r $d^{*}$ ) fur Complexe dünner Krystallblättchen abgeleitet hat, und zwar nehmen wir'den Ausdruck für die Zeit $d N$, welche eine Schwingung braucht, um ein unendlich dunnes hexagonales Packet von der Dicke $d \delta$ zu durchsetzen. Dieser Ausdruck wird lauten

$$
d N=\frac{1}{2}\left(n_{1}+n_{2}\right) d \delta,
$$

wenn $n_{1}$ und $n_{2}$ die beiden Brechungsindices der unter einander gleich genommenen Lamellen sind. Es ist ersichtlich, dass $\frac{1}{2} \int_{0}^{+1}\left(n_{1}+n_{2}\right) d \delta$ den Brechungsindex des Systemes darstellt, welches aus unendlich vielen parallel auf einander geschichteten hexagonalen Packeten besteht. Wir haben also

$$
N=\frac{1}{2}\left(n_{1}+n_{2}\right) \text {. }
$$

Wenn aber die Dichte der Aufschichtung sich ändert, und zwar so, dass $p^{\prime}$ Lamellen nach der Aenderung denselben Raum einnehmen, wie $p$ vor der Aenderung, so können wir schreiben

$$
p^{\prime} d \delta^{\prime}=p d \delta
$$

es wird also angenommen, dass die Grösse $d \delta$ bei solcher Veränderung in die $d \delta^{\prime}$ ubergeht. Jetzt werden wir statt der friberen Formel (1) eine andere schreiben mussen, und zwar

$$
d N^{\prime}=\frac{1}{2}\left(n_{1}{ }^{\prime}+n_{2}{ }^{\prime}\right) d \delta^{\prime},
$$

wo $n_{1}$ und $n_{2}$ ebenfalls ibren Werth verändert haben. Nun aber müssen die Lamellen optisch unverändert bleiben, es muss also die Beziehung bestehen

$$
\left(n_{1}{ }^{\prime}+n_{2}{ }^{\prime}\right) d \delta^{\prime}=\left(n_{1}+n_{2}\right) d \delta \text {. }
$$

Wenn wir diesen Ausdruck mit dem obigen (2) vergleichen, so bekommen wir

*) Traité de Cristallographie geométrique et pbysique $\mathbf{2}, 266 \mathrm{f}$. 
und somit wird jetzl

$$
\left(n_{1}^{\prime}+n_{2}^{\prime}\right)=\left(n_{1}+n_{2}\right) \frac{p^{\prime}}{p}
$$

$$
N^{\prime}=\frac{1}{2}\left(n_{1}+n_{2}\right) \frac{p^{\prime}}{p}=\frac{p^{\prime}}{p} N .
$$

Diese merkwürdige Beziehung behauptet, dass bei solcher Aenderung der Dichte in den Lamellencomplexen, bei welcher keine elastischen Krăfte in Thätigkeit gesetzt werden, sich die Brechungsindices der den Complex durchsetzenden Strahlen verändern, wie die Dichte der Aufschichtung, oder, was dasselbe ist, wie die linearen Dichtigkeiten des Complexes vor und nach der Aenderung.

Mit anderen Worten ändert sich diejenige Axe des inversen Ellipsoids, welche senkrecht zur Richtung der Aenderung der Dichte steht*). Diese Formel könnte man selbstverständlich auch für andere Fälle ableiten.

Bezeichnen wir ebenso, wie frther, die der Messung entnommenen Brechungsindices, resp. Axen des inversen Ellipsoids, für Kalkspalh mit $\omega$ und $\varepsilon$ und die berechneten mit $\omega^{\prime}$ und $\varepsilon^{\prime}$. Es sei die lineare Dichte nach der verticalen Axe im Aragonit $a_{1}$ und diejenige senkrecht darauf im Mittel $a_{2}$. Die entsprechenden Dichten für Kalkspath bezeichnen wir mit $c_{1}$ und $c_{2}$. Nach dem Obigen soll die Beziehung bestehen

$$
\omega=\omega^{\prime} \frac{c_{1}}{a_{1}}, \quad \varepsilon=\dot{\varepsilon}^{\prime} \frac{c_{2}}{a_{2}} .
$$

Wenn wir jetzt die Volumdichte des Aragonit mit $\Delta_{a}$ und die des Calcits mit $\Delta_{c}$ bezeichnen, so ist

$$
\Delta_{a}: \Delta_{c}=a_{1} a_{2}^{2}: c_{1} c_{2}^{2} \text {. }
$$

Tragen wir hierin die Werthe der Verhältnisse $a_{1}: c_{1}$ und $a_{2}: c_{2}$, die aus obigen Gleichungen gefunden sind, ein, so bekommen wir für das Verhältniss den Werth

$$
\Delta_{a}: \Delta_{c}=1,076 \text {, }
$$

was ziemlich nabe der Wirklichkeit entspricht, weil aus der Grösse 2,72 für die Dichte des Calcits sich diejenige des Aragonits zu 2,93 ergiebt.

Man hat also in dieser Thatsache gewisse Gründe, um die Dimorphie des betrachteten Stoffes nicht elwa durch die Verschiedenheit der Molek ale in beiden Fällen, sondern durch die verschiedene Gruppirung derselben zu erklären.

Wenn wir nun die Ergebnisse dieser Betrachtungen mit der Thatsache vergleichen, dass das Kalium-Lithiumsulfat die Polarisationsebene dreht,

*) Das entspricht der bekannten Thatsache, dass beim Zusammendrücken der Krystalle die Doppelbrechung senkrecht zur Druckrichtung zunimmt. 
also nach der Theorie von Sobncke und Mallard aus schraubenarlig gelagerten zweiaxigen Molekularschichten aufgebaut ist, so ist man einigermassen berechtigt zu behaupten, dass unter gunstigen Umständen die Verzwilligung der einzelnen Molekularnetze, welche eine 》Grenzform « besitzen, so weit vor sich gehen kann, dass homogene Krystalle von höherer Symmetrie daraus entstehen und somit eine Dimorphie zu Stande kommt. Die von anderer Seite bei der Erklärung der optischen Anomalien bestrittene Möglichkeit solcher Verzwilligung, welche zu den Gestalten des regulären Systems führt, wird durch Bildung von Verwachsungen, wie sie das S. 592 besprochene Berylliumsulfat zeigt, veranschaulicht.

Ich balte es für eine angenehme Pflicht, hier öffentlich meinen herzlichen Dank Herrn Professor P. Groth auszusprechen, welcher mich im Laufe meiner Arbeit so freundlich mit Wort und That unterstutzt hat. 